\title{
Establishing the National Chlamydia Screening Programme in England: results from the first full year of screening
}

\author{
D S LaMontagne, K A Fenton, S Randall, S Anderson, P Carter, on behalf of the \\ National Chlamydia Screening Steering Group
}

See end of article for authors' affiliations

Correspondence to: D Scott LaMontagne, MPH, FRIPH, CS, Health Protection Agency, Communicable Disease Surveillance Centre, 61 Colindale Avenue, London NW9 5EQ, UK; scott. lamontagne@hpa.org.uk

Accepted for publication 13 August 2004

\begin{abstract}
Background: The phased implementation of the National Chlamydia Screening Programme (NCSP) began in September 2002. The NCSP offers opportunistic screening for chlamydia to women and men under 25 years of age attending clinical and non-clinical screening venues using non-invasive urine or vulvo-vaginal swab samples tested via nucleic acid amplification. This review describes the implementation of the NCSP, reports positivity rates for the first year, and explores risk factors for genital chlamydial infection.

Methods: Cross sectional study of the first year's screening data from the NCSP. A standardised core dataset for each screening test was collected from 302 screening venues, excluding genitourinary medicine (GUM) clinics, across 10 phase 1 programme areas. We estimated chlamydia positivity by demographic and behavioural characteristics, and investigated factors associated with infection through univariate and multivariate analyses.

Results: Chlamydia positivity among people under 25 years of age screened in non-GUM settings was $10.1 \%(1538 / 15241)$ in women and $13.3 \%(156 / 1172)$ in men. Risk factors varied by sex: for womenage 16-19, non-white ethnicity, and sexual behaviours were associated with infection; for men-only age 20-24 and non-white ethnicity were associated with infection.

Discussion: In the first phase of the NCSP, 16413 opportunistic screens among young adults under 25 years of age were performed at non-GUM settings and testing volume increased over time. Rates of disease were similar to those found during the English screening pilot and were comparable to the first year of widespread screening in Sweden and the United States. The screening programme in England will continue to expand as further phases are included, with national coverage anticipated by 2008.
\end{abstract}

$\mathrm{T}$ he most commonly reported bacterial sexually transmitted infection (STI) in the England is Chlamydia trachomatis, ${ }^{1}$ with serious sequelae in untreated infected women-for example, chronic pelvic pain, pelvic inflammatory disease (PID), ectopic pregnancy, and infertility. ${ }^{2}$ Complications among men with untreated infection include urethritis, epididymitis, and Reiter's syndrome. ${ }^{2}$ Recent evidence has also suggested that infection can cause male infertility. ${ }^{3}$ Since a high proportion of chlamydial infections are asymptomatic, ${ }^{2}$ screening programmes have evolved to detect and treat individuals with prevalent undiagnosed infections and their partners. ${ }^{4-7}$ These programmes have reported reductions in prevalence ${ }^{6-10}$ and incident PID $^{11} 12$ after implementation. Other studies have demonstrated that screening for genital chlamydial infection is both cost beneficial and cost effective..$^{13-15}$

In England, a comprehensive review of the evidence justifying screening for genital chlamydial infection against the Wilson-Jungner criteria ${ }^{16}$ was published by the chief medical officer's (CMO) expert advisory group on Chlamydia trachomatis. ${ }^{17}$ The principal conclusion from this review was a call to establish a national chlamydia screening programme, focusing on young women attending clinical settings who were at risk of infection. ${ }^{17}$ This call was strengthened through the inclusion of chlamydia screening in the action plan of the National Strategy for Sexual Health and HIV, which aims to reduce the transmission and prevalence of STIs. ${ }^{18}$

In 1998, the Department of Health (DoH) in England funded a pilot of an opportunistic screening programme to explore how best to implement chlamydia screening. The investigators of the chlamydia pilot concluded it was feasible and acceptable to opportunistically screen asymptomatic women 16-24 years of age attending different healthcare settings, including general practices, contraceptive clinics, young people's services, women's services (for example, termination, gynaecology, and antenatal), and genitourinary medicine (GUM) clinics. ${ }^{19}$ The screening pilot also reported high rates of disease, on average $10 \%$ prevalence among young women attending general practice and other healthcare settings. ${ }^{20}$ Based on this evidence and with the guidance from the lessons learned in the screening pilot, the DoH agreed funding for a phased implementation of the National Chlamydia Screening Programme (NCSP) for England. This paper overviews the structure and process of the NCSP, reports positivity rates by demographic and behavioural characteristics, and explores risk factors for chlamydia from the first reporting year (1 April 2003-31 March 2004) of opportunistic screening for phase 1 programmes.

\section{METHODS}

\section{Programme overview}

The goal of the NCSP in England is to control genital chlamydial infection through the early detection and treatment of asymptomatic infections and prevention of sequelae and onward transmission. Funding and national leadership

Abbreviations: CDSC, Communicable Disease Surveillance Centre; GUM, genitourinary medicine; LCR, ligase chain reaction; NAATs, nucleic acid amplification tests; NCSP, National Chlamydia Screening Programme; PCR, polymerase chain reaction; PCTs, primary care trusts; PIAG, patient information advisory group; PID, pelvic inflammatory disease; SDA, strand displacement amplification; TMA, transcription mediated amplification 
are provided by the DoH, with scientific support from the Health Protection Agency Communicable Disease Surveillance Centre (CDSC). The programme is guided by a national chlamydia screening steering group (NCSSG), comprising multidisciplinary representation from relevant clinical and public health bodies engaged in sexual health, including GUM, contraception and family planning, obstetrics and gynaecology, nursing, health advising, general practice, and microbiology. This body advises the DoH on clinical and laboratory protocols and procedures for implementing opportunistic screening in primary health care settings-for example, contraception clinics, young people's services, general practices, etc. Screening protocols for the national programme are contained in a core requirements document, and are disseminated to local programme areas to standardise local screening activity. ${ }^{21}$ Phased implementation of the NCSP began with 10 programme areas selected in September 2002 for phase $1 . .^{22}$ Programme areas are composed of consortia of primary care trusts (PCTs), which are the geographic and service boundaries of the National Health Service (NHS). In January 2004, the second phase of the NCSP began with an additional 16 programme areas encompassing a further 54 PCTs. This brings current population coverage of the NCSP to an estimated $30 \%$ of all sexually active young people aged 15-24 years in England.

Local programme areas implement screening activities guided by the national core requirements. Administrative structures vary locally but usually include a local multidisciplinary steering group, chlamydia screening office and coordinator, programme lead, and clinical staffing, in partnership with PCTs, local laboratories, and healthcare providers.

All local screening activity is coordinated by a local chlamydia screening coordinator, working out of a designated chlamydia screening office. A multidisciplinary local CSSG oversees the local programme implementation and is responsible for ensuring that data are reported to the DoH. Mandatory guidelines on the structure, process, and outcome monitoring for local programmes have been produced by the national CSSG and are contained in a core requirements document (available at www.dh.gov.uk). Also contained within the core requirements document are the Patient Information Advisory Group (PIAG) approved standard screening dataset to be collected by all screening sites. The core data items are transferred electronically to the Health Protection Agency, Colindale, on a quarterly basis for national programme monitoring. Local programmes have some flexibility to adapt their screening activities to reflect local need. All programmes areas are developing locally relevant materials to complement nationally available screening resources; some areas are examining the feasibility of screening chlamydia positive patients for gonorrhoea; and other areas plan to include additional screening venues-for example, local prisons, in their activities. All such enhancements are funded locally. As the programme is still in its infancy, locally driven research and evaluation will needed to inform unresolved operational issues-for example, screening intervals and engaging men. ${ }^{23}$

The target population for screening is young men and women under the age of 25 years who are attending healthcare facilities not traditionally associated with providing specialist sexual health services. This approach expands the number of locations (that is, screening venues) that young people can attend which are offering chlamydia screening as part of their services. These include contraceptive clinics, general practices, young people's services, antenatal services, colposcopy and infertility units, and termination of pregnancy clinics. Screening is also encouraged to those within the target age group through innovative outreach strategies, such as "pee in a pot" days at military bases, university campuses or health fairs, mobile vans or buses for contact with young people, prisons, and other nontraditional settings. People routinely attending GUM clinics are already tested for Chlamydia trachomatis as a part of standardised clinical protocols, and as such are not the primary target for the "opportunistic" nature of this national programme. People falling within the screening guidelines are offered a chlamydia test when attending a venue participating in the programme, regardless of the reason for their attendance. The attendance, itself, is the "opportunity" created to educate and encourage the uptake of screening for chlamydia. People under 16 years of age are offered screening if they are determined by the test initiator to be "Frazer competent." ${ }^{24}$ Every person offered screening receives a detailed patient information leaflet which summarises screening procedures and management outcomes. Implied consent is acquired by client self completion of a test request form and provision of a clinical specimen for testing.

Non-invasive samples, principally urine and self collected vulvo-vaginal swabs, are submitted to centralised local laboratories and tested using one of three common nucleic acid amplification tests (NAATs) ${ }^{*}$ - polymerase chain reaction (PCR) Amplicor or Cobas Amplicor (Roche Diagnostics, Basel, Switzerland), strand displacement amplification (SDA) BDProbeTec (Becton, Dickinson and Company, Franklin Lakes, NJ, USA), or transcription mediated amplification (TMA) Aptima Combo 2 Assay (Gen-Probe Incorporated, San Diego, CA, USA), per the manufacturer's instructions. All positive and equivocal samples are confirmed, either through testing using a different NAAT or rerun of the sample using the same platform. ${ }^{21}$

All clients are notified of test results, based on their preferred method, such as letter, telephone call, or text message. People testing positive are contacted up to three times for treatment and partner notification. Treatment is provided in accordance with published guidelines, ${ }^{25}$ although directly observed azithromycin ( $\mathrm{g}$ oral tablets) is the preferred option. Alternative regimens are prescribed, as clinically necessary. ${ }^{25}$ Clients receive treatment at no charge, which may be dispensed at the original testing venue, by the local chlamydia screening coordinator, via referral to a GUM clinic, or other method as negotiated by the client. All people testing positive, and especially those who exhibit symptoms suggestive of chlamydial infection, are offered the opportunity to attend a GUM clinic for further STI testing. Routine tests of cure are not performed, unless the patient has been treated with erythromycin or there are serious concerns about treatment compliance.

Patients are offered the choice of notifying their own partners (patient referral), or supplying information for the health adviser or local chlamydia coordinator to notify the partner, without the patient's name being given (provider referral). Partner notification activities are also undertaken by various trained personnel-for example, the health adviser, chlamydia coordinator, staff based at a GUM clinic, programme lead, and/or test initiator; according to national standards. ${ }^{26}$ Prophylactic treatment to partners is provided free of charge. All documentation relating to treatment of index patient and follow up of partner notification activities is collated locally at the central chlamydia screening office for local audit and is reported annually in aggregate summary to the DoH.

*Even though the Abbott LCx test (Abbott Laboratories, Abbott Park, IL, USA) was withdrawn from the UK market in early 2003, one programme area in phase 1 tested specimens via ligase chain reaction (LCR) from April to August 2003 because of an overstock of available test kits and reagents. 
Standardised information about the demographic and behavioural characteristics of the population screened, location of screening, laboratory test method used, and test result is collected uniformly across all local programme areas by the use of a test request form and is reported in disaggregate nationally to CDSC. Data reporting to CDSC is approved by the PIAG.

\section{Sample selection}

In this paper, tests reported to CDSC by 15 June 2004 are included. Of those, we selected only tests performed outside of GUM clinics and which were taken for opportunistic screening purposes only, as they most closely reflect the efforts to extend chlamydia testing services to people who might not normally have been tested and who potentially represent a "hidden reservoir" of asymptomatic infections within the young adult population. Tests were categorised as opportunistic screening if the reason for the test was for screening purposes. Tests performed for diagnostic reasons or because a client was a contact of a chlamydia positive were not included. An additional $4 \%(n=674)$ of tests were excluded from analysis because of unknown or missing data for test result, sex, age, type of test, or inconsistent sample type (for example, male tests with self collected vulvo-vaginal swabs).

\section{Data analysis}

We performed cross sectional descriptive analyses of the population tested and assessment of factors associated with infection. Distributions of demographic, behavioural, and testing characteristics within the population were tabulated. $\chi^{2}$ tests and univariate odds ratios were calculated. Multivariate analyses were performed using logistic regression to explore the inter-relation of factors associated with infection within the male and female populations separately, as we hypothesised that the factors associated with infection between the two populations might be different. ${ }^{2}$

We used chlamydia positivity, rather than prevalence, as the dependent variable in our univariate and multivariate analyses, as studies have shown that positivity is a valid surrogate measure of prevalence. ${ }^{27}{ }^{28}$ Further, since this is a largely naive population to screening and the testing period is 12 months or less, the likelihood of repeat testers is minimal and would not have an appreciable impact on our estimates.

All data analysis was performed in SPSS 12.0 for Windows (SPSS Inc, Chicago, IL, USA) with two tailed significance levels of $\mathrm{p}<0.05$.

\section{RESULTS}

Opportunistic screening in phase 1 of the NCSP occurred in a staged approach with the number of programme areas and the number of venues within each programme area offering chlamydia screening increasing from the first quarter of

Table 1 Number of programme areas and venues* offering opportunistic screening for Chlamydia trachomatis by quarter, National Chlamydia Screening Programme in England, 1 April 2003-31 March 2004

\begin{tabular}{lll}
\hline Quarter & $\begin{array}{l}\text { Number of programme } \\
\text { areas }\end{array}$ & $\begin{array}{l}\text { Number of screening } \\
\text { venues }\end{array}$ \\
\hline 1st, Apr-Jun 2003 & 4 & 74 \\
2nd, Jul-Sep 2003 & 5 & 121 \\
3rd, Oct-Dec 2003 & 9 & 184 \\
4th, Jan-Mar 2004 & 10 & 247 \\
Total & 10 & 302 \\
\hline *Does not include GUM clinics.
\end{tabular}

operations to the last (table 1). For example, in the first quarter of the NCSP, four programme areas were offering screening at 74 venues outside of GUM clinics; by the end of the first full year of reporting, almost 250 non-GUM venues across all 10 phase 1 programme areas were offering opportunistic screening to young people. A total of 16413 opportunistic screening tests were performed outside GUM settings during the first full year of the NCSP. Following the phased approach, over 1000 chlamydia screens $(6 \%$ of the total) were done in the first quarter, and the last quarter of the first year accounted for almost $50 \%$ of all screening tests (table 2).

We found $10.1 \%$ positivity among women less than 25 years of age opportunistically screened at settings outside of GUM clinics in the first year of the NCSP (table 2). The population of women screened was primarily white and tested at contraceptive clinics. Over half of the female population was under 20 and the other half $20-24$ years of age. Behavioural risks among women were common: $44 \%$ of the reporting population indicated a new sex partner in the last 3 months and/or two or more sex partners in the last year. SDA and PCR were the most commonly used diagnostic platforms in local laboratories. Urine was the most common specimen type, but nearly $30 \%$ of tests were done on self collected vulvo-vaginal swabs.

We found $13.3 \%$ positivity among men less than 25 years of age opportunistically screened at settings outside of GUM clinics in the first year of the NCSP (table 2). The population of men screened was primarily white, with $45 \%$ of tests done at contraceptive clinics and $26 \%$ done at colleges and universities. The male population was slightly younger than the female population-62\% under 20 years of age. Behavioural risks among men were more common: $56 \%$ of the reporting population indicated a new sex partner in the last 3 months and $60 \%$ reported two or more sex partners in the past year. Urine was collected from all men.

Women ages $16-19$ years were $43 \%$ more likely to test positive for chlamydia than those 20-24 years old (table 2). Women of black Caribbean ethnicity were nearly twice as likely to test positive. Behavioural risks were also associated with infection in women, even after controlling for covariates. Women tested with SDA were more likely to test positive and this factor also held after multivariate adjustment (table 2).

Among men, the groups more likely to test positive were somewhat different from those for women (table 2). After controlling for all factors in multivariate analysis, slightly older males, those 20-24 years of age, were more than twice as likely as those under 20 to test positive. Similar to women, black Caribbean or mixed ethnicity males were also more than twice as likely to be infected. Among men, behavioural risks were not statistically associated with an increased risk of infection, either in unadjusted or adjusted analyses (table 2). Additionally, men screened at colleges and universities and at young people's clinics had a reduced likelihood of infection than those tested in contraceptive clinics. Like their female counterparts, men screened using the SDA test had a significantly higher likelihood of testing positive (table 2).

To understand why higher positivity was found with the SDA test, an exploratory analysis was performed, dividing the population into those tested with SDA and those tested using another NAAT. Groups with higher positivity tended to be over-represented in the population tested with SDA (data not shown). For example, there were larger proportions of black and mixed ethnic groups and people reporting a new sex partner among people tested with SDA $(\mathrm{p}<0.05)$, and these groups were almost twice as likely to test positive for chlamydia than white people or those who did not report a 


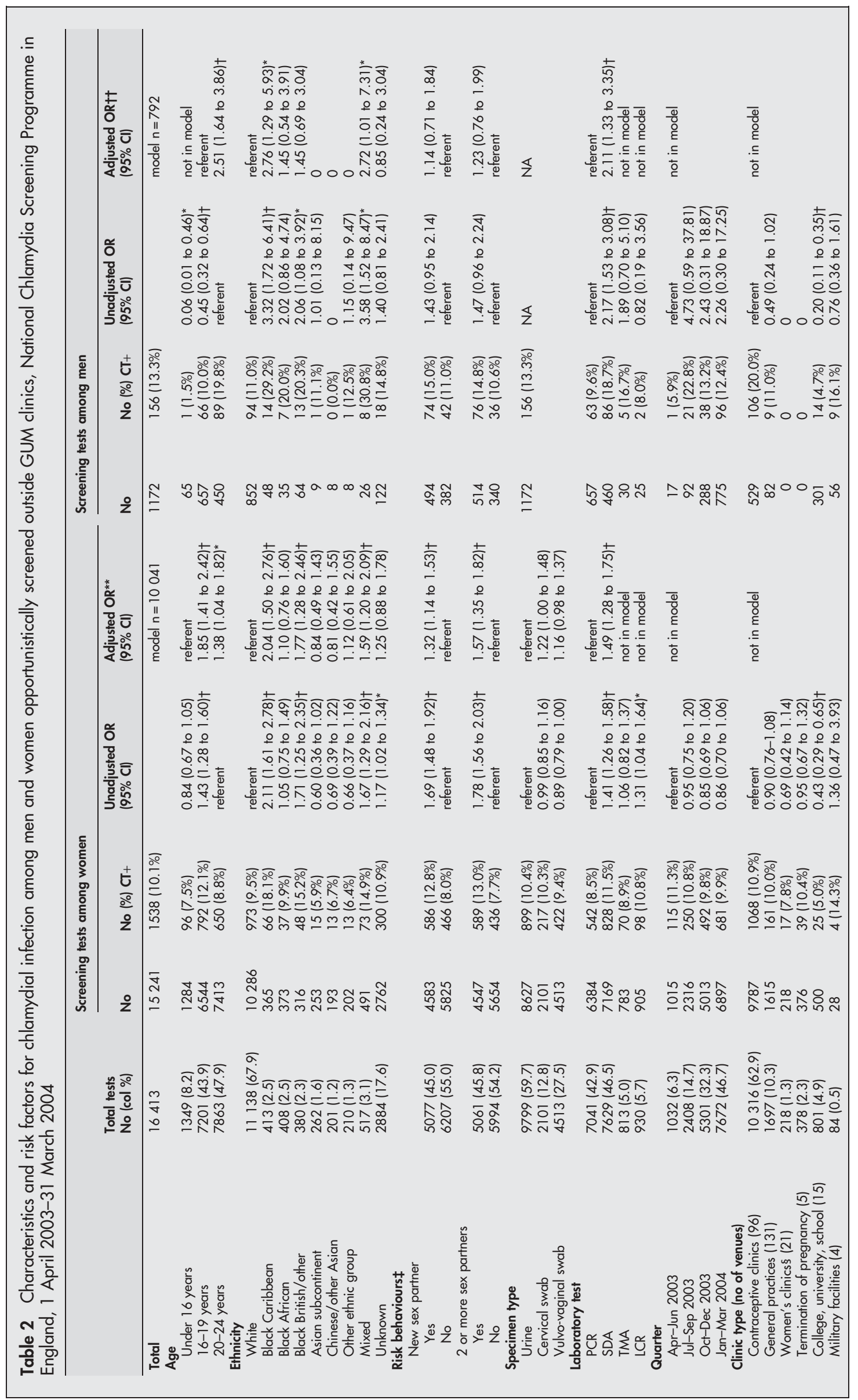




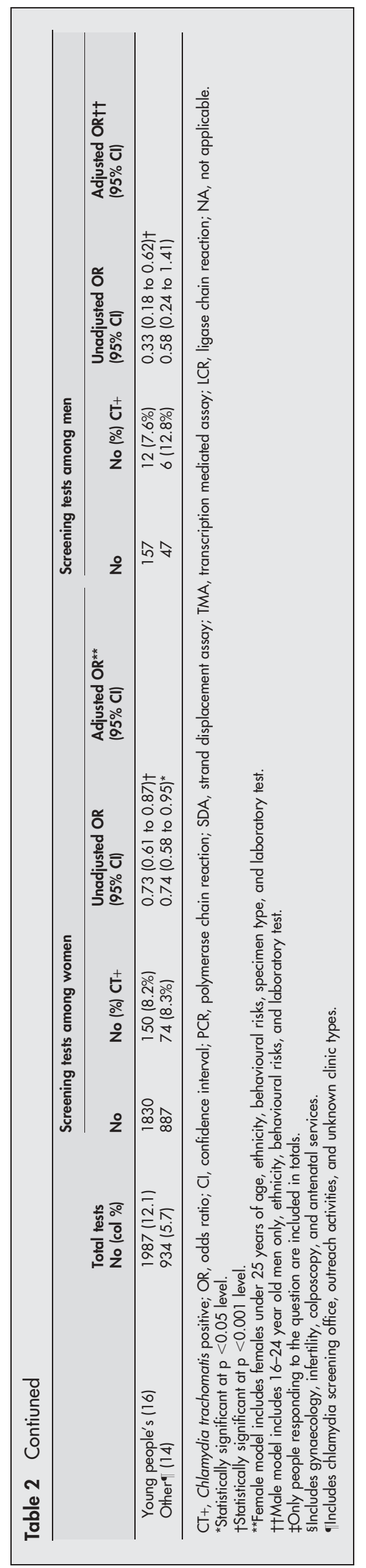

new sex partner in the last 3 months (table 2). Further, there were higher concentrations of 20-24 year old females in the population tested with LCR, PCR, and TMA and the positivity in this group was less than 16-19 year olds.

\section{DISCUSSION}

The phased implementation of the National Chlamydia Screening Programme in England has begun. The number of phase 1 programme areas, the number of venues within programme areas, and the total number of screens performed all increased over time, reflecting our phased approach. Because the programme has just begun, it is important to place in context the screening volume and its impact on coverage. Economic models have shown that one of the most critical aspects to ensure the success of a widespread screening programme is uptake. ${ }^{14}{ }^{1529-31}$ Recent estimates from the United States by Levine and colleagues suggest that screening coverage was highest in areas that experienced reductions in prevalence after several years of aggressive and comprehensive screening. ${ }^{32}$ Data from the first few years of routine chlamydia testing in Sweden also reflect the impact of high screening volumes. ${ }^{9}$ Continued efforts to increase screening coverage in England are focused not only on expanding the number of programme areas involved, but also increasing the volume of testing at the screening venues within those programme areas. This will be monitored closely as the NCSP continues to expand.

The first year of screening has also detected similar levels of infection among people consenting to be screened as was found in the original chlamydia screening pilot. ${ }^{20}$ Screening programmes in other countries reported chlamydia prevalences ranging from $6 \%$ in Sweden ${ }^{9}$ to $12 \%$ in the north western region of the United States ${ }^{63}$ in their first year of implementation. Although those same programmes screened a larger number of women than thus far accomplished in England, the similar rates of infection at the start affirms that the opportunistic approach-selectively screening those thought to be at higher risk-has proved to be a successful strategy in disease detection. The data from the first year of the NCSP justify our continued focus on young women and men attending healthcare settings as performed in the original pilot.

Another unique outcome of the first year results is the demonstration that opportunistic screening can and does occur in a wide variety of settings. Encouragingly, the second highest volumes of screening were from 131 general practices (in five of the programme areas) and 16 young people's services (in six programme areas), both of which are not traditionally centred around sexual health service provision. Much has been made recently about the ability or willingness of GPs to become involved in the NCSP. Oakeshott et al suggest in a recent article that without remuneration GPs would not only not participate in screening but also that the programme would not succeed..$^{34}$ Unlike the research pilot of opportunistic chlamydia screening in which GPs were paid on a per test basis, ${ }^{19}$ the NCSP funding in phase 1 did not include the same payments to GPs (as it is not a research project). The NCSP first year data seem to suggest that GPs are willing to offer chlamydia screening to their clientele without reimbursement incentives from the NCSP. Over 10\% of all screening tests were done within general practice, and that proportion increased throughout the first year and continues to do so in preliminary data from the first quarter of the second year (data not shown). We are encouraged by these numbers, as strong efforts have been made by both the national management team, as well as local chlamydia screening coordinators and their teams, to engage primary care and to ease the implementation of screening in those settings. Creative delivery strategies utilised in phase 1 
programme areas address some of the barriers to screening within general practice, ${ }^{35}$ and include: (1) allowing patients to self select for screening and self complete the test request form (saving time); (2) training of practice nurses to make appropriate invitations for screening (reducing the need for expensive medical consultant involvement); (3) covering administrative time for specimen and data collection (augmenting costs); (4) shifting the responsibility for notification of results and follow up to a local chlamydia screening office (reducing workload burden within general practice); and (5) empowering GPs to holistically attend to the physical and sexual health needs of their young adult population (enhancing the skills and capabilities of general practice staff). The lessons learnt about implementing screening in primary care from the first phase of the programme have informed the development of guidelines for chlamydia screening in general practice as well as model local enhanced service contracts outlining set standards and outcomes for screening in this setting. The devolved nature of general practice provision in England means that efforts to encourage local involvement of GPs in chlamydia screening will become a major challenge of the programme in future years. Indeed, phase 2, and subsequent phase 3, implementation areas will encourage more widespread primary care involvement than previous phases.

Data from the NCSP's first year confirm that the epidemiological profile of both the men and women screened is nearly identical to that found in numerous studies in the United Kingdom ${ }^{17}{ }^{176}$ and in Europe, ${ }^{37}$ with highest chlamydia positivity among women 16-19 years of age and men 2024. The age related differences in chlamydia positivity between women and men screened was expected given the results of other studies. ${ }^{1933} 36$ Additionally, people who have acquired a sex partner recently or who have had several sex partners were at increased risk of infection. However, the association between behavioural risks and infection among men did not reach statistical significance in univariate analysis or multivariate modelling. This could be the result of a small sample size for men (less than 1200 male tests reported), an under-reporting of sexual risk taking among the female population, or an actual difference in the sexual behaviour of men versus women. There is evidence from the Natsal study of sexual behaviour in Britain to support a behavioural difference between men and women: men reported a greater number of lifetime and recent sex partners, as well as more frequent partner change, than women. ${ }^{38}$

The sexual behaviour data reported through the NCSP have provided additional benefit by further refining our analysis of risk behaviours that are associated with people testing positive for chlamydia. This will allow us to better understand the behavioural components contributing to the spread of STIs, ${ }^{39}$ and monitor behavioural changes in the population that may affect our disease control efforts. Other established STI surveillance networks, principally through the statutory KC60 returns from GUM clinics, do not collect sexual behaviour data. The collection of these data in the NCSP is the first large scale programme targeting sexual health to include behavioural surveillance. The use of this information allows us an additional tool in prevention efforts to address, and eventually arrest, the observed increases in STIs in England.

The noted increase positivity among those tested with the SDA platform is an unexpected mystery. The concentration of higher risk people in the SDA tested population and lower risk people in the population tested using other NAATs might help explain the variations in positivity between the four different nucleic acid amplification tests. However, interlaboratory variation in the use of the testing platforms, adjusted sensitivity and specificity of each NAAT in a "real

\section{Key messages}

- The phased implementation of the National Chlamydia Screening Programme has begun: 16413 chlamydia screens to young men and women during the first year confirm the feasibility of opportunistic screening in nonGUM settings

- The NCSP has demonstrated $10 \%$ positivity among women and $13 \%$ positivity among men opportunistically screened; this is similar to the findings from the original screening pilot and affirms the opportunistic approach is a successful strategy for disease detection

- $30 \%$ of specimens collected from women were self taken vulvo-vaginal swabs (VVS), demonstrating the feasibility and acceptability of VVS as an alternative to urine collection for women

- The NCSP is the first national sexual health initiative to include routine behavioural surveillance, which improves our understanding of the behavioural factors driving STIs and enhances our ability to design appropriate prevention messages

world" setting, or the utilisation of lower cut-off threshold for positive confirmation within the laboratory could also influence the performance and outcome of the SDA test. It would be worthwhile to further analyse this difference. The DoH has recently funded the Microbiological Diagnostic Assessment Service to carry out a comparative evaluation of the sensitivity, specificity and performance of PCR, TMA, and SDA in three laboratories in England (Department of Health, personal communication). This evaluation may also provide additional context for explaining our findings.

Finally, researchers have suggested that targeted annual screening of 15-24 year old females, combined with treating $50 \%$ of partners of chlamydia positive females and increasing condom use, could dramatically reduce the prevalence of chlamydia in the population. ${ }^{29}$ The NCSP includes dedicated funding and guidelines for a strong partner follow up activities in local programme areas ${ }^{21}$ to ensure women do not become re-infected from an untreated partner and that partners of positive women do not continue to spread infection to others. The NCSP provides for the testing and free treatment of all sexual contacts, regardless of age, through application of rigorous national partner notification standards. ${ }^{26}$ In future years, the marrying of partner notification data with screening volume and coverage data will provide an enhanced summary of the NCSP's impact on the population. We hope to expedite disease reductions through the combined approach of screening and comprehensive treatment and follow up.

It is clear from our results that we have some way to go to demonstrate reductions in disease similar to what was experienced in Sweden and the United States; however, the data from this first year of screening are encouraging. New lessons are being learnt on the process and outcomes of opportunistic screening and methods for enhancing its implementation in a diverse range of healthcare settings. Insights are being gained into the best methods for engaging men in sexual health; innovation in treatment and partner notification in sites outside of GUM clinics; and sharing of information on best practice across the breadth of the health service system in England. The collection of an expanded and disaggregate sexual health dataset is improving our understanding of the distribution and determinants of genital chlamydial infection. The NCSP will undoubtedly continue to 
make inroads into the prevention and control of this infection in England.

\section{ACKNOWLEDGEMENTS}

Funding is provided by the Department of Health (England), London. The opinions of the authors do not necessarily represent the views of the Department of Health (England).

For all their efforts in bringing chlamydia screening to their communities, the authors would like to thank the clinical, laboratory and administrative personnel from the phase 1 programme areas: Camden and Islington, Cornwall, Hull and East Riding, Lambeth/ Southwark/Lewisham, Leeds, Nottingham, Portsmouth, Southend on Sea, the Wirral, and York.

\section{CONTRIBUTORS}

DSL is the lead scientist for the NCSP, collated, extracted and analysed the data, and wrote the paper; KAF is the chair of the National Chlamydia Screening Steering Group; SR is the national medical adviser of the NCSP; SA and PC are the national coordinators of the NCSP; each provided additions to the manuscript as coauthors.

\section{Authors' affiliations}

D S LaMontagne, K A Fenton, HIV and Sexually Transmitted Infections Department, Health Protection Agency, Communicable Disease Surveillance Centre, 61 Colindale Avenue, London NW9 5EQ, UK S Randall, Department of Health, Sexual Health and Substance Misuse Team, Room 580D, Skipton House, 80 London Road, London, and Ella Gordon Unit, St Mary's Hospital, Portsmouth PO3 6AD, UK

S Anderson, P Carter, Department of Health, Sexual Health and Substance Misuse Team, Room 580D, Skipton House, 80 London Road, London SE1 6LH, UK

Competing interest: All authors declare no competing interests in the preparation or submission of this manuscript.

\section{REFERENCES}

1 Health Protection Agency, SCIEH, ISD, National Public Health Service for Wales, CDSC Northern Ireland, and UASSG. Renewing the focus. HIV and other sexually transmitted infections in the United Kingdom in 2002. London: Health Protection Agency, 2003.

2 Stamm WE. Chlamdyia trachomatis infections of the adult. In: Holmes KK Sparling PF, Mardh PA, et al, eds. Sexually transmitted diseases, 3rd ed. United States: McGraw-Hill Health Professions Division, 1999:407-22.

3 Idahl A, Boman J, Kumlin U, et al. Demonstration of Chlamydia trachomatis lgG antibodies in the male partner of the infertile couple is correlated with a reduced likelihood of achieving pregnancy. Hum Reprod 2004;19:1 121-6.

4 Mausner JS, Kramer S. Epidemiology-an introductory text, 2nd ed. Philadelphia: WB Saunders, 1985.

5 Handsfield HH, Jasman LL, Roberts PL, et al. Criteria for selective screening for Chlamydia trachomatis infection in women attending family planning clinics. JAMA 1986;255:1730-4.

6 Britton TF, Delisle S, Fine D. STDs and family planning clinics: a regional program for chlamydia control that works. Am J Gynecol Health 1992;6:80-7.

7 Addiss DG, Vaughn ML, Hillis SD, et al. History and features of the Wisconsin Chlamydia trachomatis control program. Fam Plan Perspect 1994;26:83-9.

8 Addiss DG, Vaughn ML, Ludka D, et al. Decreased prevalence in Chlamydia trachomatis infection associated with a selective screening program of family planning clinics in Wisconsin. Sex Transm Dis 1993;20:28-35.

9 Herrmann B, Egger M. Genital Chlamydia trachomatis infections in Uppsala County, Sweden, 1985-1993: declining rates for how much longer? Sex Transm Dis 1995;22:253-60.

10 Mertz KJ, Levine WC, Mosure DJ, et al. Trends in the prevalence of chlamydial infections - the impact of community-wide testing. Sex Transm Dis 1997;24:169-75.

11 Scholes D, Stergachis A, Heidrich FE, et al. Prevention of pelvic inflammatory disease by screening for cervical chlamydial infections. N Engl J Med 1996;334:1362-6.
12 Ostergaard L, Andersen B, Moeller JK, et al. Home sampling versus conventional swab samples for screening of Chlamydia trachomatis in women: a cluster-randomized 1-year follow-up study. Clin Infect Dis 2000;31:951-7.

13 Marrazzo JM, Celum CL, Hillis SD, et al. Performance and cost-effectiveness of selective screening criteria for Chlamydia trachomatis infection in women: implications for a national chlamydia control strategy. Sex Transm Dis 1997;24:131-41.

14 Paavonen J, Puolakkainen M, Paukku M, et al. Cost-benefit analysis of firstvoid urine Chlamydia trachomatis screening program. Obstet Gynecol 1998;92:292-8.

15 Kretzschmar $M$, Welte $R$, van den Hoek $A$, et al. Comparative model-based analysis of screening programs for Chlamydia trachomatis infections. Am J Epidemiol 2001;153:90-101.

16 Wilson JMG, Jungner G. Principals and practice of screening for disease. Geneva: World Health Organization, 1968.

17 Chief Medical Officer's Expert Advisory Group. Main report of the CMO's Expert Advisory Group on Chlamydia trachomatis. London: Department of Health, 1998

18 Department of Health. The national strategy for sexual health and HIV. London: DoH, 2001.

19 Pimenta JM, Catchpole M, Rogers PA, et al. Opportunistic screening for genital chlamydial infection I: acceptability of urine testing in primary and secondary healthcare settings. Sex Transm Infect 2003;79:16-21.

20 Pimenta JM, Catchpole M, Rogers PA, et al. Opportunistic screening for genital chlamydial infection II: prevalence among healthcare attenders, outcome, and evaluation of positive cases. Sex Transm Infect 2003;79:22-7.

21 Department of Health. National chlamydia screening programme in England-programme overview, core requirements and data collection, 2nd ed. London: DoH, 2004.

22 Fenton KA. National chlamydia screening programme in England: the first steps. Sex Transm Infect 2004;80:000-00.

23 Department of Health. The national chlamydia screening programme in England. Programme overview, core requirements and data collection. London: DoH, Issue 2. April 2004.

24 Gillick v West Norfolk, Wisbech AHA [1986] AC1 12, [1985] 3 WLR830, [1985] 3 All ER 402 HL.

25 Clinical Effectiveness Workgroup (AGUM and MSSVD). Clinical effectiveness guideline for the management of Chlamydia trachomatis genital tract infection. London: British Association for Sexual Health and HIV, 2002.

26 Society of Sexual Health Advisers. The manual for sexual health advisers. London: Society of Sexual Health Advisers, 2004.

27 Dicker LW, Mosure DJ, Levine WC. Chlamydia positivity versus prevalence. What's the difference? Sex Transm Dis 1998;25:251-3.

28 LaMontagne DS, Fenton KA, Pimenta JM, et al. Using chlamydia positivity to estimate prevalence: evidence from the Chlamydia Screening Pilot in England. Int J STD AIDS 2004; (in press)

29 Welte R, Kretzschmar M, Keidl R, et al. Cost-effectiveness of screening programmes for Chlamydia trachomatis. Sex Transm Dis 2000;27:518-29.

30 Kretzschmar M, van Duynhoven YTHP, Severijnen AJ. Modeling prevention strategies for gonorrhea and chlamydia using stochastic network simulations. Am J Epidemiol 1996; 144:306-17.

31 Honey E, Augood C, Templeton A, et al. Cost effectiveness of screening for Chlamydia trachomatis: a review of published studies. Sex Transm Infect 2002;78:406-12.

32 Levine WC, Dicker LW, Devine $O$, et al. Indirect estimation of chlamydia screening coverage using public health surveillance data. Am J Epidemiol 2004;160:91-6.

33 Centers for Disease Control and Prevention. Sexually transmitted disease surveillance 2002 Supplement: chlamydia prevalence monitoring project annual report 2002. Atlanta: CDC, Division of STD Prevention, October 2003.

34 Oakeshott $P$, Hay $P$, Pakianathan $M$. Chlamydia screening in primary care. Br J Gen Pract 2004;54:491-3.

35 McNulty CAM, Freeman E, Bowen J, et al. Barriers to opportunistic chlamydia testing in primary care. Br J Gen Pract 2004;54:508-14.

36 Adams EJ, Charlett A, Edmunds WJ, et al. Chlamydia trachomatis in the United Kingdom: a systematic review and analysis of prevalence studies. Sex Transm Infect 2004; (in press).

37 Wilson JS, Honey E, Templeton A, et al. A systematic review of the prevalence of Chlamydia trachomatis among European women. Hum Reprod Update 2002;8:385-94.

38 Fenton K, Korovessis C, Johnson AM, et al. Sexual behaviour in Britain: reported sexually transmitted infections and prevalent genital Chlamydia trachomatis infection. Lancet $2001 ; 358: 1851-4$

39 Garnett GP. The geographical and temporal evaluation of sexually transmitted disease epidemics. Sex Transm Infect 2002;78(Suppl 1):114-19. 